\title{
Enhanced Positioning Technique with QR Calibration
}

\author{
A.S. Ja’afar
}

\begin{abstract}
This paper describes an integrated positioning system that can be used for both indoor and outdoor navigation. The GPS (or any other system) in many scenarios is not sufficiently accurate especially for indoor. Even at a fixed location the accuracy is slightly changing with time due to signal propagation environment condition where current parameters do not reflect this. To address this, we propose a new criteria call "accumulated accuracy". With this new parameter the GPS accuracy is estimated in a practical way using real data. In this paper, a sliding window technique is proposed for improving "accumulated accuracy" and it show improvement. In addition to this, the $Q R$ calibration is introduced for further improving the "accumulated accuracy". This technique can also be applicable to various other positioning techniques.
\end{abstract}

Keywords - Positioning, Navigation, Indoor Positioning.

\section{Introduction}

GPS system is widely used by public as the main navigation solution. It is based on a principle of continuous monitoring of signals from the multiple dedicated satellites (at least 4) which are used for determining the user position for navigation purposes [1][2]. However due to environment conditions, the GPS signal can be blocked, reflected, refracted and this could affect the accuracy of user position [2][3][4]. Because of this, at a fixed location the positioning accuracy is always inconsistent with time or epoch where current parameters do not represent accurately. Recently it has been shown by multiple authors [5][6][7] that navigation systems relying solely on GPS are not ubiquitous and have significant constrains in many life critical scenarios. Therefore, a technique called "Hybrid Navigation" has been suggested as a mean for improving availability and accuracy of conventional navigation systems- In this case positioning decision is made based on a signals received from more than 1 source and the more accurate position is achieved through collaborative processing power of multiple signals. However, even recently introduced hybrid technique suffer from high accumulating error in positioning determination. In our work we define a new parameter, termed "accumulated accuracy" and we demonstrate improvements in the performance of legacy systems estimated with this parameters. Improvement to the "accumulated accuracy" of GPS positioning will enhance accuracy of indoor positioning and this will further improve the efficiency of navigation process.

We also propose sliding window techniques for improving the "accumulated accuracy" as mentioned before. Three different type of sliding window is introduced and results show improvement. To further improve the "accumulated accuracy", a novel approach to hybrid

\section{A. S. Ja'afar}

School of Computing and Communication, Lancaster University United Kingdom positioning where a QR code is used as external calibration. In this paper, we demonstrate the hybrid GPS+QR calibration on the same scenario as "sliding window" but future research will concentrate on mobile users. Our finding, that this technique not only specific for GPS positioning, but is applicable to other positioning techniques.

The rest of the paper is structured as follows: vision based positioning is outline in section II; Global Positioning System algorithm is highlighted in section III; hybrid GPS + QR code positioning is elaborated in section IV, simulation result and discussion are described in section V; conclusion and future work in section VI.

\section{Vision Based Positioning}

The basic algorithm of localization include the time-ofarrival (TOA), angle-of-arrival (AOA), time-different-ofarrival (TDOA), received signal strength based (RSS), and fingerprint techniques [1][5]. To enhance the accuracy of positioning, hybrid measurement and data fusion based on these basic techniques have been developed by researchers around the globe with utilizing certain features in dedicated network-based positioning such as WiMAX[8][9][10].

The smartphone nowadays loaded with GNSS module, communication modules and sensors give it massive potential for pedestrian navigation or positioning system. The potential of smartphone positioning or navigation system include the conventional GNSS positioning, Wi-Fi fingerprint technique, Bluetooth, Visual positioning, pedestrian map matching and dead reckoning, cellular positioning system, magnetic anomaly matching, FM radio positioning and others [2][6].

As suggested by [7], the mobile positioning system potential can be focus on movement-based positioning and vision-based positioning (VBP) which estimated through dead reckoning (DR), inertial navigation (IN), and optical image sensor. Among the advantages of the mobile positioning system are better accuracy up to few centimetres and more reliable whenever wireless signal cannot penetrate the building. Others positioning system that have been done which utilize the optical sensor include the estimation position on the hallway without depending on database [11]. The achievement was less than $1 \mathrm{~m}$ but constrain only for indoor hallway. Mobile Visual Indoor Positioning System (MoVIPS) [12] utilize SURF algorithm, use matching features technique on the database server to determine the user location. Besides, WLAN positioning was implemented on MOVIPS to help improving ambiguity dataset selection. W. Bejuri [13] also have done a research positioning on the hallway which combine the hybridization of WLAN and optical images. Their work are based on inverse intensity chromaticity space in order to extract feature detection on information interest point and floor edges. Again WLAN 
positioning information is used to reduce the possibly hallways selected.

The used of Quick Response code or QR code is quite common at the moment. It was first develop in Japan to solve tracking components in automotive industry. It became popular since it offers fast readability and greater storage capacity modes in numeric, alphanumeric, binary and kanji. The QR code gain wider range of usage from commercial tracking, ticketing besides as labelling. Some get used it in more innovative way such as a library with QR code which direct user to download required information, place a giant QR code advertisement on tops of building so it can be view through Google Maps. Researchers from RMIT University and La Trobe University have use the active RFID assisted by QR Code for sighted and blind pedestrian navigation in building [14]. RFID tags are places at entrance, corners and selected points to help determine the user current location. While QR code is places along with RFID tags at entrance and selected interest points to help the sighted in giving information for navigation purposes.

\section{Global Positioning System}

GPS is an established and widely used by civilian for positioning, navigation and other applications. At least 4 out of 76 GPS satellites are needed to observe at the same time to determine the position. Pseudorange measurement technique usually adopted to estimate the 3D position in ECEF coordinate position before convert into WGS84 format (longitude and longitude). To solve the pseudorange equation from at least from 4 satellites which is non-linear, it have to be linearized using Taylor's series expansion [3][4][15].

The pseudorange measurement equation is given by the range of satellites position $\left(x_{i}, y_{i}, z_{i}\right)$ to the user position $\left(x_{u}, y_{u}, z_{u}\right)$ with bias user clock error $b_{u}$. Figure 1 show at least 4 satellites pseudorange observation at the same time needed in order to determine user location.

$$
\rho_{i}=\sqrt{\left(x_{i}-x_{u}\right)^{2}+\left(y_{i}-y_{u}\right)^{2}+\left(z_{i}-z_{u}\right)^{2}}+b_{u}
$$

Where $\mathrm{i}$ are number of satellites.

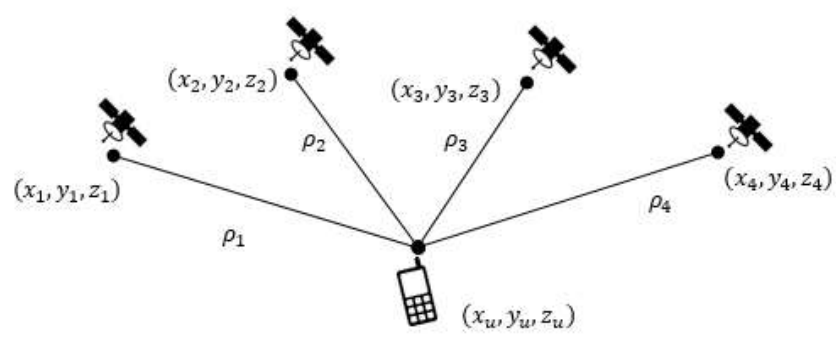

Figure 1: GPS stand-alone positioning

Based on Figure 1 and equation 1, the pseudorange observation and satellites position are known while user position $\left(x_{u}, y_{u}, z_{u}\right)$ and clock error bias $b_{u}$ are unknowns. In this case, to solve four unknown parameters it required minimum of four pseudorange observation from satellites in order to determine the user position. Differentiate the pseudorange basic equation

$$
\begin{gathered}
\delta \rho_{i}=\frac{\left(x_{i}-x_{u}\right) \delta x_{u}+\left(y_{i}-y_{u}\right) \delta y_{u}+\left(z_{i}-z_{u}\right) \delta z_{u}}{\sqrt{\left(x_{1}-x_{u}\right)^{2}+\left(y_{1}-y_{u}\right)^{2}+\left(z_{1}-z_{u}\right)^{2}}}+\delta b_{u} \\
\delta \rho_{i}=\frac{\left(x_{i}-x_{u}\right) \delta x_{u}+\left(y_{i}-y_{u}\right) \delta y_{u}+\left(z_{i}-z_{u}\right) \delta z_{u}}{\rho_{i}-b_{u}}+\delta b_{u}
\end{gathered}
$$

The initial value of user position $\left(x_{u}, y_{u}, z_{u}\right)$ can be set to centre of the earth $(0,0,0)$, while the unknowns $\delta x_{u}, \delta y_{u}$, $\delta z_{u}, \delta b_{u}$ can be calculated. These unknown values will correct the new user position $\left(x_{u}, y_{u}, z_{u}\right)$ and the process is done iterative until the value of unknowns $\delta x_{u}, \delta y_{u}$, $\delta z_{u}, \delta b_{u}$ are smaller than threshold value. The equation in matrix form

$$
\left[\begin{array}{l}
\delta \rho_{1} \\
\delta \rho_{2} \\
\delta \rho_{3} \\
\delta \rho_{4}
\end{array}\right]=\left[\begin{array}{llll}
\alpha_{11} & \alpha_{12} & \alpha_{13} & 1 \\
\alpha_{21} & \alpha_{22} & \alpha_{23} & 1 \\
\alpha_{31} & \alpha_{32} & \alpha_{33} & 1 \\
\alpha_{41} & \alpha_{42} & \alpha_{43} & 1
\end{array}\right]\left[\begin{array}{l}
\delta x_{u} \\
\delta y_{u} \\
\delta z_{u} \\
\delta b_{u}
\end{array}\right]
$$

Where

$$
\begin{aligned}
\alpha_{i 1} & =\frac{x_{i}-x_{u}}{\rho_{i}-b_{u}} \\
\alpha_{i 2} & =\frac{y_{i}-y_{u}}{\rho_{i}-b_{u}} \\
\alpha_{i 3} & =\frac{z_{i}-z_{u}}{\rho_{i}-b_{u}}
\end{aligned}
$$

We can rearrange the matrix become:

$$
\left[\begin{array}{l}
\delta x_{u} \\
\delta y_{u} \\
\delta z_{u} \\
\delta b_{u}
\end{array}\right]=\left[\begin{array}{llll}
\alpha_{11} & \alpha_{12} & \alpha_{13} & 1 \\
\alpha_{21} & \alpha_{22} & \alpha_{23} & 1 \\
\alpha_{31} & \alpha_{32} & \alpha_{33} & 1 \\
\alpha_{41} & \alpha_{42} & \alpha_{43} & 1
\end{array}\right]^{-1}\left[\begin{array}{l}
\delta \rho_{1} \\
\delta \rho_{2} \\
\delta \rho_{3} \\
\delta \rho_{4}
\end{array}\right]
$$

The $\delta_{x}$ can be found through least squares approach.

$$
\delta_{x}=\left[\begin{array}{ll}
\alpha^{T} & \alpha
\end{array}\right]^{-1} \alpha^{T} \delta_{\rho}
$$

Figure 2 shows the output result of the 25 epochs positioning calculation relative to the exact location for $\mathrm{X}, \mathrm{Y}$ and $\mathrm{Z}$ axis through the least squares approach. Here, the interest will be more on $\mathrm{X}$ and $\mathrm{Y}$ as this will determine the latitude and longitude in position. From the graph, the relative $\mathrm{Y}$ give more fluctuation output result between positive to negative compare to relative output $\mathrm{X}$, which started on more to negative region then the margin error fluctuate on the positive side. The modulus of total area for relative $\mathrm{X}$, relative $\mathrm{Y}$ and relative $\mathrm{Z}$ are 34.1540, 87.2081 and 248.3674 . 
Proc. of the Second Intl. Conf. on Advances in Information Processing and Communication Technology - IPCT 2015 Copyright (c) Institute of Research Engineers and Doctors, USA .All rights reserved.

ISBN: 978-1-63248-044-6 doi: 10.15224/ 978-1-63248-044-6-119
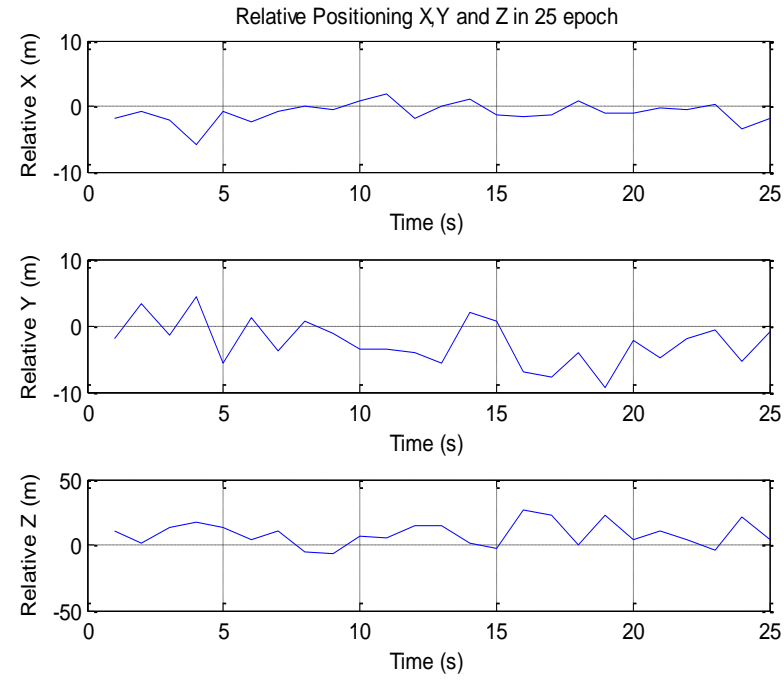

Figure 2: Relative position of $\mathrm{X}, \mathrm{Y}$ and $\mathrm{Z}$ position for 25 epochs

In order to evaluate accuracy of GPS over a time interval, we propose new criteria called "accumulated accuracy":

$$
A=\frac{1}{T} \int_{0}^{T} \sqrt{\left(f(t)-f_{o}(t)\right)^{2}} d t
$$

Where $f(t)$ is the relative positioning graph under epoch period

$f_{o}(t)$ is the base line of zero error

$T \quad$ is the period of accumulated epoch

\section{Iv. Hybrid GPS + QR Positioning}

In this paper, we will focus on GPS as the main positioning system, assisted by QR barcode as the visual based positioning. This is after consideration of the optical sensor is commonly available at mobile phone right now. The hybrid of GPS and visual based positioning (QR barcode) of our proposed system give several advantages:

i) Cost effective: both GPS and optical sensor modules are available on the mobile phone, so no need to implement new infrastructure to enhance the user positioning. The QR code does not need any license to use it.

ii) Reliability: visual based positioning give higher accuracy can be used as the assisted or correction to the common GPS especially in NLOS environment which give higher error location.

iii) Fast processing: no need to access the server database, as the algorithm processing will be done straightforward.

The QR barcode can store data in several encoding modes include byte, numeric, alphanumeric and kanji. It has greater storage capacity compare to standard 2D barcode and equipped with Reed Solomon code error correction which make it robust to interpret. We proposed QR barcode as the assisted to GPS algorithm positioning calculation where the QR code tags can be install on several important part of building such as the entrance, point of interest and boundary/corner of the building. The information of the exact location where the QR code is located is encoded on the QR code itself so the QR code will act as the error location correction or calibration to the GPS calculation. The example of QR code is depicted in Figure 3.

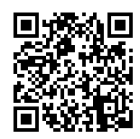

Figure 3: Encrypted QR code

The block diagram of the hybrid positioning of GPS and QR code are shown in Figure 4.

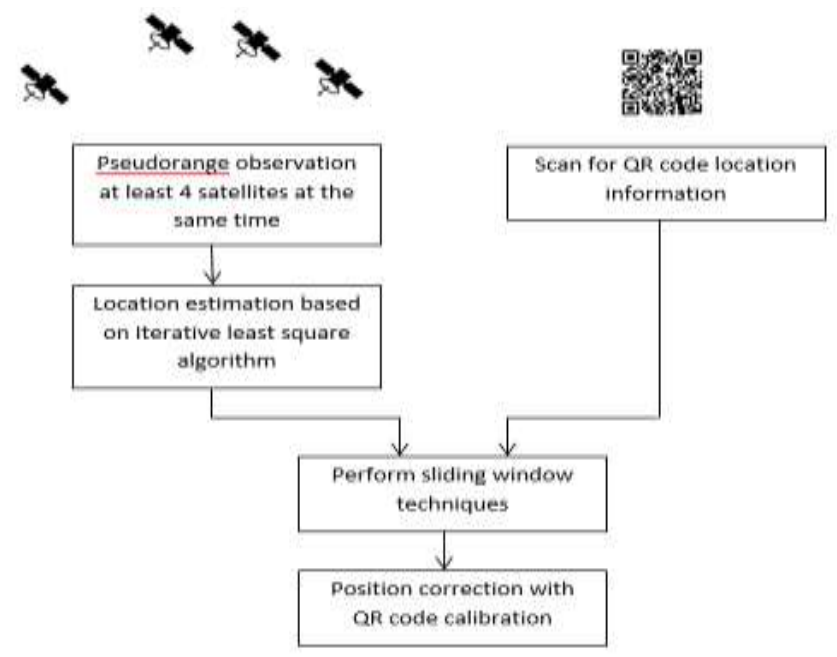

Figure 4: Block diagram of hybrid GPS and QR Code calibration

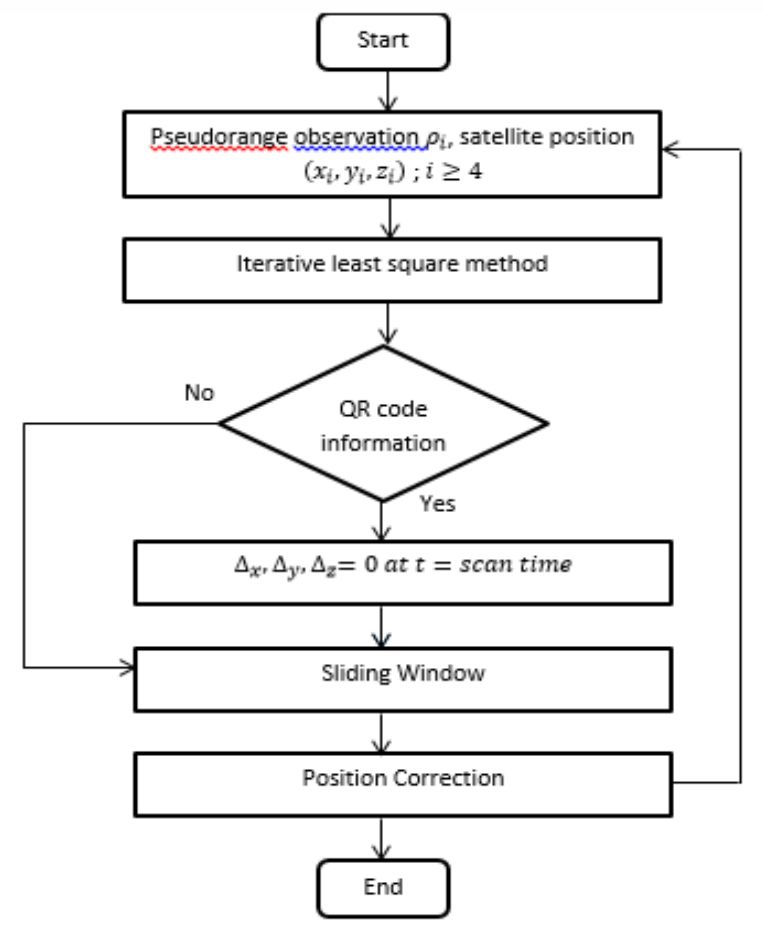

Figure 5: Flowchart of hybrid system 
Proc. of the Second Intl. Conf. on Advances in Information Processing and Communication Technology - IPCT 2015

Copyright ( $($ Institute of Research Engineers and Doctors, USA .All rights reserved.

ISBN: 978-1-63248-044-6 doi: 10.15224/ 978-1-63248-044-6-119

\section{v. Simulation Results}

The iterative least square algorithm was written in MATLAB script with the important two input parameter which are the pseudorange measurement and satellite position. The calculation is done in Earth Centered Earth Fixed Coordinate System (ECEF) before it can be converted in World Geodetic System (WGS84) format. The user position is fixed at certain location and positioning calculation totally based on pseudorange measurement and satellites position.

We introduce three different kind sliding window technique to smooth the changeability in determine the user position. The first sliding window technique (\#1) will be based on previous location if the next relative position is greater than the average sliding window of the past relative value. The sliding window that we introduce is depicted in general equation below.

$$
S_{i}=\frac{1}{W} \sum_{j=i-w}^{i-1} S_{j}
$$

where

$\mathrm{S}$ is the relative position of $\mathrm{X}, \mathrm{Y}$, and $\mathrm{Z}$ in ECEF format $\mathrm{W}$ is the sliding window size

The graph in Figure 6 shows the relative to exact position of different size of sliding window (\#1) based on the actual result in Figure 2.
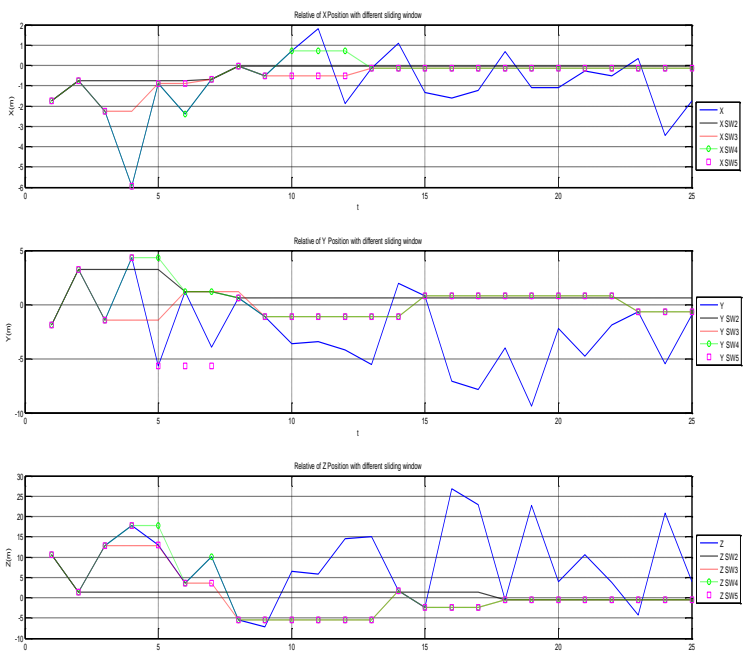

Figure 6: Relative position of different size of sliding window (SW) technique \#1 from 2 s to $5 \mathrm{~s}$

The maximum size of sliding window chosen here is up to $5 \mathrm{~s}$, where we believe the higher number than that figure make it less practical in application. Based on the graph in Figure 6, it is clearly show the improvement in accuracy for sliding window size from $2 \mathrm{~s}$ to $5 \mathrm{~s}$. Table 1 shows the ratio of accumulated accuracy based on the graph in Figure 6 with different size of sliding window. It is clearly show that the overall minimum number of this sliding window technique give better accuracy.
TABLE 1: RATIO OF ACCUMULATED ACCURACY WITH DIFFERENT SIZE OF SLIDING WINDOW

\begin{tabular}{|c|c|c|c|r|}
\hline Graph (Relative) & SW 2 & SW 3 & SW 4 & SW 5 \\
\hline X & 19.34 & 38.75 & 55.63 & 49.51 \\
\hline Y & 32.16 & 31.98 & 37.98 & 49.85 \\
\hline Z & 14.79 & 42.01 & 48.60 & 44.00 \\
\hline
\end{tabular}

Figure 7 depicts the result of relative in output position $\mathrm{X}, \mathrm{Y}$ and $\mathrm{Z}$ for different size of the second type of sliding window technique. The second type of sliding window where for each epoch will be based on total average of past relative position. If the next relative errors are greater than the average number of past sliding window, it will base on the average value of the past reading.
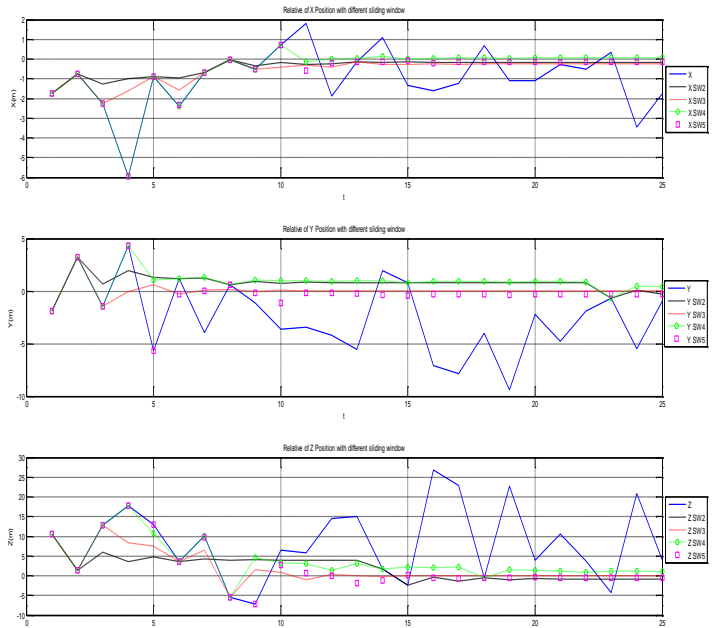

Figure 7: Relative position of different size of sliding window (SW) \#2 from $2 \mathrm{~s}$ to $5 \mathrm{~s}$

Table 2 describes the calculation of result under the curve (modulus) of different size of sliding window (\#2) condition. From the data in Table 2, it clearly show that the sliding window with size 3 give the overall best result in total across the relative $\mathrm{X}, \mathrm{Y}$ and $\mathrm{Z}$ with 83.7.

TABLE 2: RATIO OF ACCUMULATED ACCURACY WITH DIFFERENT SIZE OF SLIDING WINDOW

\begin{tabular}{|c|c|c|c|c|}
\hline $\begin{array}{c}\text { Graph } \\
\text { (Relative) }\end{array}$ & SW 2 & SW 3 & SW 4 & SW 5 \\
\hline X & 30.47 & 42.07 & 49.23 & 54.21 \\
\hline Y & 28.10 & 9.85 & 33.96 & 26.62 \\
\hline Z & 28.25 & 24.43 & 42.35 & 37.57 \\
\hline
\end{tabular}

The third sliding window condition, for every specific time of position will depend on average of the past sliding window and average of the future time of the sliding window. The result of the third technique is depicted in Figure 7. Each of the sliding window size improves the accuracy as the figure shown in Table 3. However this technique consume in time because every specific time it have to repeat the calculation of advance in time depending on size of the sliding window chosen. 
Proc. of the Second Intl. Conf. on Advances in Information Processing and Communication Technology - IPCT 2015

Copyright (C) Institute of Research Engineers and Doctors, USA .All rights reserved.

ISBN: 978-1-63248-044-6 doi: 10.15224/ 978-1-63248-044-6-119
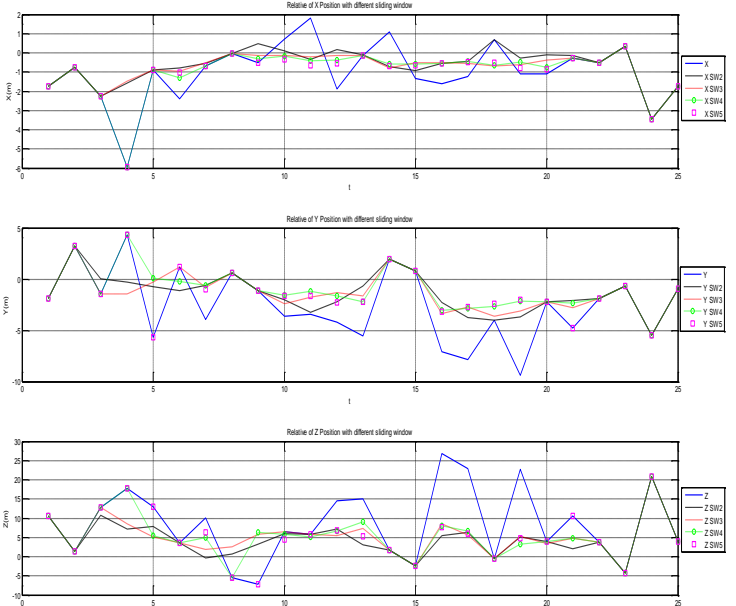

Figure 8: Relative position of different size of sliding window sliding window (SW) \#3 from $2 \mathrm{~s}$ to $5 \mathrm{~s}$

Even though this technique gives some improvement in accuracy, the increment of sliding window size doesn't give much improvement as can see in Table 3. The improvement is only by $\sim 50 \%$.

\begin{tabular}{|c|c|c|c|c|}
\hline $\begin{array}{c}\text { Graph } \\
\text { (Relative) }\end{array}$ & SW 2 & SW 3 & SW 4 & SW 5 \\
\hline$X$ & 57.73 & 57.79 & 74.11 & 77.26 \\
\hline Y & 54.41 & 55.76 & 54.21 & 65.61 \\
\hline Z & 51.42 & 57.48 & 64.00 & 68.78 \\
\hline
\end{tabular}

Table 4 describes the total ratio of the three different sliding window techniques with different window size. The first sliding window technique with size of 2 seconds gives the best result with smallest ratio of 19.34. Based on total ratio in Table 4, it can be conclude that the second type of sliding window give more consistent through out of different sliding window size compare to others sliding window technique.

TABLE 4: RATIO OF ACCUMULATED ACCURACY WITH DIFFERENT SIZES AND SLIDING WINDOW TECHNIQUES

\section{Sliding}

Window

\begin{tabular}{|c|c|c|c|c|c|}
\hline Technique & Graph & SW 2 & SW 3 & SW 4 & SW 5 \\
\hline \multirow{2}{*}{$\# 1$} & X & 19.34 & 38.75 & 55.63 & 49.51 \\
\hline & Y & 32.16 & 31.98 & 37.98 & 49.85 \\
\hline & Z & 14.79 & 42.01 & 48.60 & 44.00 \\
\hline \multirow{2}{*}{ Total ratio } & 66.29 & 112.74 & 142.21 & 143.36 \\
\hline \multirow{2}{*}{ \# } & X & 30.47 & 42.07 & 49.23 & 54.21 \\
\hline & Z & 28.10 & 9.85 & 33.96 & 26.62 \\
\hline & Total ratio & 86.82 & 76.35 & 125.54 & 118.4 \\
\hline
\end{tabular}

\begin{tabular}{|c|c|c|c|c|c|}
\hline & $\mathrm{X}$ & 57.73 & 57.79 & 74.11 & 77.26 \\
\hline \multirow{2}{*}{$\mathbf{Y} 3$} & 54.41 & 55.76 & 54.21 & 65.61 \\
\hline & $\mathrm{Z}$ & 51.42 & 57.48 & 64.00 & 68.78 \\
\hline & Total ratio & 163.56 & 171.03 & 192.32 & 211.65 \\
\hline
\end{tabular}

The improvements achieved through the introduction of "sliding window" could be further enhanced by incorporating calibration over $\mathrm{QR}$ at certain a priori given positions. Assume at certain scenario (preferably indoor, where GPS accuracy is not sufficient) a user standing at QR code tag location. The user will tag to the $\mathrm{QR}$ code which the information of exact location will be known by the user. In this case, the velocity will be equal to zero before the information transfer will be processes which navigate to the certain destination. The GPS positioning location based on pseudorange is calculated for each epoch, so in this scenario the relative error is forced to the zero. Here, the location error is assume equal at 0 at the time equal to 0 and 11 for $\mathrm{X}$ position, and time equal to 0 and 13 for $\mathrm{Y}$ position. The simulation is done based on three different kind of sliding window with different size. The result is shown in Figure 9 to 11 .
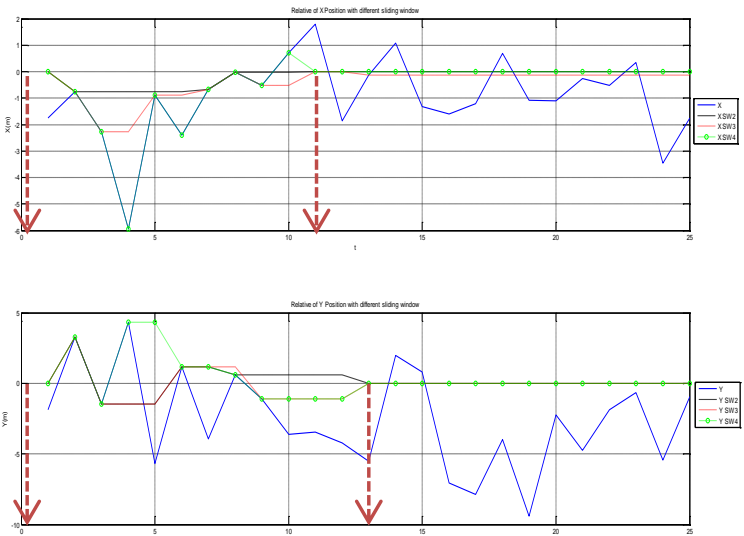

Figure 9: Relative position sliding window (SW) \#1, window size from $2 \mathrm{~s}$ to $4 \mathrm{~s}$
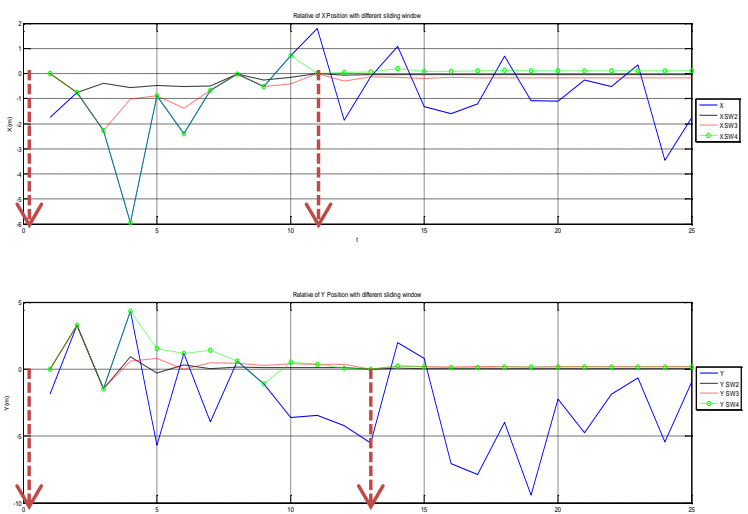

Figure 10: Relative position sliding window (SW) \#1, window size from $2 \mathrm{~s}$ to $4 \mathrm{~s}$ 
Proc. of the Second Intl. Conf. on Advances in Information Processing and Communication Technology - IPCT 2015

Copyright (C) Institute of Research Engineers and Doctors, USA .All rights reserved.

ISBN: 978-1-63248-044-6 doi: 10.15224/ 978-1-63248-044-6-119
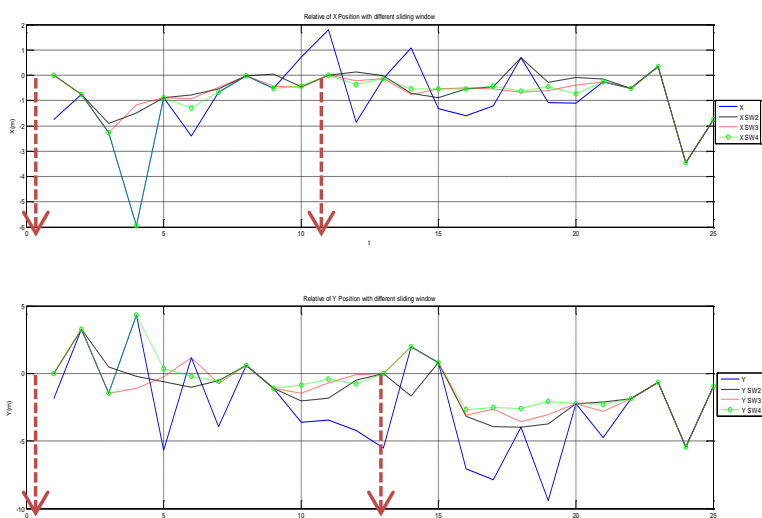

Figure 11: Relative position sliding window (SW) \#1, window size from 2s to $4 \mathrm{~s}$

Table 5 show the ratio figure of each sliding window technique with different window size based on result in Figure 9-11. Based on this ratio, it clearly show that the overall second sliding window technique give the best result particularly on sliding window size of 2 and 3 . The improvement of the accuracy in this case is nearly $90 \%$. The third sliding window technique still show improvement but it does not good enough.

TABLE 5: RATIO OF ACCUMULATED ACCURACY, DIFFERENT WINDOW SIZES AND SLIDING WINDOW TECHNIQUES, WITH QR CODE CALIBRATION

\section{Sliding}

Window

\begin{tabular}{lll|l} 
Technique Graph & SW 2 & SW 3 & SW 4
\end{tabular}

\begin{tabular}{|l|l|l|l|l|l|} 
& $\mathrm{X}$ & 13.2872 & 30.6251 & 41.5023 & 41.3878 \\
\hline & $\mathrm{Y}$ & 14.8406 & 17.8426 & 23.8415 & 35.7038 \\
\hline & $\mathrm{X}$ & 12.5833 & 30.2828 & 45.7340 & 42.0003 \\
\hline \#2 & $\mathrm{Y}$ & 8.4727 & 12.1854 & 20.2894 & 26.0947 \\
\hline & $\mathrm{X}$ & 49.4138 & 52.8743 & 68.7671 & 69.8656 \\
\hline & $\mathrm{Y}$ & 49.0886 & 46.9745 & 45.9086 & 58.5245 \\
\hline
\end{tabular}

\section{vi. Conclusion}

In this paper, the propose a new parameter, termed "accumulated accuracy" in positioning and show that accumulated accuracy of conventional iterative least square approach based on pseudorange observation is not sufficient in certain conditions. We propose a novel "sliding window" technique for improving the accumulated accuracy and compare three different types of sliding windows technique. Different sizes of sliding windows up to 5 epochs with 3 types of sliding window were investigated. All the results are shown in ratio to the total area under the curve. The best ratio is the first technique sliding window with the size of 2 epochs. In order to achieve further improvement, we combine this conventional GPS algorithm positioning calculation with the visual based positioning, in this case hybrid with the QR code as external calibration point. This is taken in consideration the mobile phone nowadays is equipped with optical sensors. The hybrid GPS and QR code calibration improve the accuracy for all 3 different type of sliding window. However the second type sliding window gives more consistent in the result analysis.

\section{Acknowledgment}

The first author appreciate Universiti Teknikal Malaysia Melaka (UTeM) and Ministry of Education Malaysia for their sponsor and support. The author also would like to thank Prof Garik Markarian and Dr Leila Musavian for their great help, valuable comments and suggestions.

\section{References}

[1] S. A. Zekavat, R. M. Buehrer, Handbook of Position Location Theory, Practice and Advances, John Wiley \& Sons, IEEE PRESS, 2011.

[2] P. D. Groves, Principles Of GNSS, Inertial, and Multisensor Integrated Navigation Systems, Artech House, 2008.

[3] E. D. Kaplan, C. J. Hegarty, Undertsanding GPS, Principles and Applications, $2^{\text {nd }}$ Ed. Artech House, 2006.

[4] J. B. Tsui, Fundamentals of Global Positioning System Receivers, A Software Approach, $2^{\text {nd }}$ Ed. John Wiley \& Sons, 2005.

[5] J. Figueiras, S. Frattasi, Mobile Positioning and Tracking From Conventional to Cooperative Techniques, John Wiley \& Sons, 2010.

[6] P. D. Groves, L. Wang, D. Walter, H. Martin, K. Voutsis, and Z. Jiang, "The four key challenges of advanced multisensor navigation and positioning," 2014 IEEE/ION Position, Locat. Navig. Symp. PLANS 2014, no. May, pp. 773-792, May 2014.

[7] D. Liu, B. Sheng, F. Hou, Rao, and H. Liu, "From Wireless Positioning to Mobile Positioning: An Overview of Recent Advances," IEEE Syst. J., pp. 1-11, 2014.

[8] A. A. M. Isa, G. Markarian, K.A. Noordin, "Wireless Location Positioning Based on WiMAX Features - A Preliminary Study," Mobile Lightweight Wireless Systems, pp 254-262, 2009.

[9] A. A M. Isa, G. Markarian, "Positioning Technologies in Wireless Broadband Communications" Cranfield Multi-Strand Conference (CMC2008), May 2008.

[10] A. Zvikhachevskaya, V. Gourov, A. A. Isa, L. Mihaylova, and G. Markarian, "Enhanced Positioning Techniques for Hybrid Wireless Networks," in INFORMATIK, 2011.

[11] H. Lee, J. Kim, S. Lee, S. Lee, and T. Lee, "Vision-based absolute indoor point positioning in the hallway without image database," 2013 IEEE Int. Conf. Consum. Electron., no. 3, pp. 284-285, Jan. 2013.

[12] M. Werner, M. Kessel, and C. Marouane, "Indoor positioning using smartphone camera," Int. Conf. Indoor Position. Indoor Navig., pp. 16, Sep. 2011.

[13] W. M. Y. Wan Bejuri, M. M. Mohamad, M. Sapri, and M. A. Rosly, "Investigation of Color Constancy for Ubiquitous Wireless LAN/Camera Positioning: An Initial Outcome," Int. J. Adv. Comput. Technol., vol. 4, no. 7, pp. 269-280, Apr. 2012.

[14] S. Alghamdi, R. Van Schyndel, and A. Alahmadi, "Indoor Navigational Aid Using Active RFID and QR-Code For sighted and Blind People," in 2013 IEEE Eight International Conference on Intelligent Sensors, Sensor Networks and Information Processing, pp. 18-22, 2013.

[15] A. Tian, D. Dong, D. Ning, and C. Fu, "GPS Single Point Positioning Algorithm Based on Least Squares," 2013 Sixth Int. Symp. Comput. Intell. Des., no. 4, pp. 16-19, Oct. 2013.

About Author (s)

\begin{tabular}{|c|c|}
\hline $\begin{array}{r}\text { A Shukur is currently a PhD candidate at } \\
\text { the School of Computing and } \\
\text { Communication, Lancaster University, } \\
\text { United Kingdom. His research topic focus } \\
\text { on indoor/outdoor positioning and } \\
\text { navigation system integration. }\end{array}$ \\
\hline
\end{tabular}

